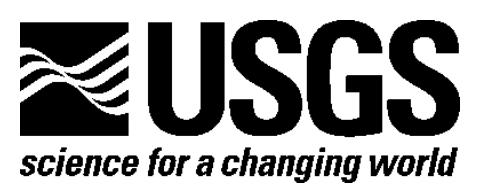

\title{
Superposed Epoch Analysis and Storm Statistics from 25 Years of the Global Geomagnetic Disturbance Index, USGS-Dst
}

Open-File Report 2012-1167

U.S. Department of the Interior U.S. Geological Survey 


\section{Superposed Epoch Analysis and Storm Statistics from 25 Years of the Global Geomagnetic Disturbance Index, USGS-Dst}

By J.L. Gannon

Open-File Report 2012-1167

U.S. Department of the Interior

U.S. Geological Survey 


\section{U.S. Department of the Interior \\ KEN SALAZAR, Secretary}

\section{U.S. Geological Survey Marcia K. McNutt, Director}

U.S. Geological Survey, Reston, Virginia: 2012

For more information on the USGS—-the Federal source for science about the Earth, its natural and living resources, natural hazards, and the environment-visit http://www.usgs.gov or call 1-888-ASK-USGS

For an overview of USGS information products, including maps, imagery, and publications, visit http://www.usgs.gov/pubprod

To order this and other USGS information products, visit $h$ ttp://store.usgs.gov

Suggested citation:

Gannon, J.L., 2012, Superposed epoch analysis and storm statistics from 25 years of the global geomagnetic disturbance index, USGS-Dst: U.S. Geological Survey Open-File Report 2012-1167, 15 p.

Any use of trade, product, or firm names is for descriptive purposes only and does not imply endorsement by the U.S. Government.

Although this report is in the public domain, permission must be secured from the individual copyright owners to reproduce any copyrighted material contained within this report. 


\section{Contents}

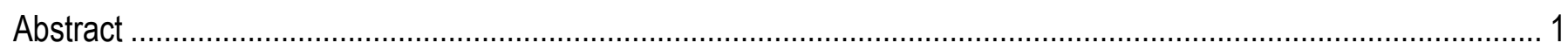

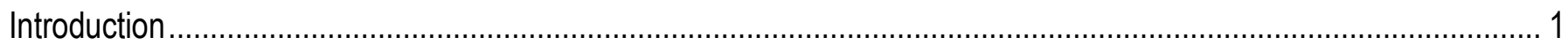

Data

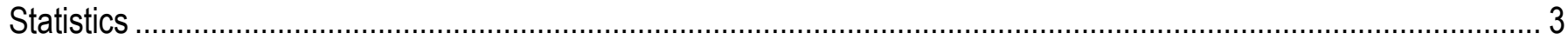

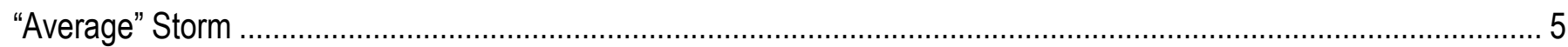

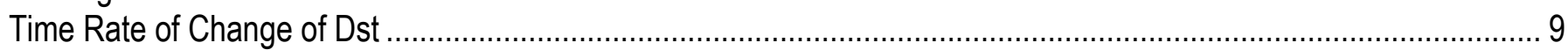

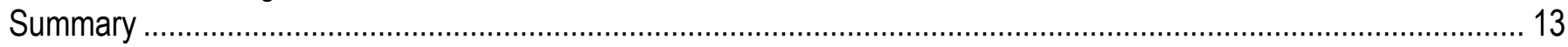

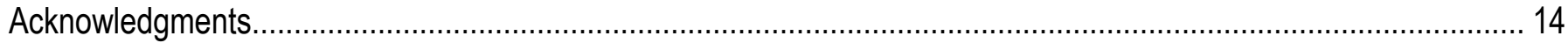

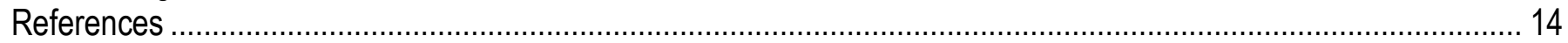

\section{Figures}

1. Graph showing an example of a storm period selected under the criteria of this study.

2. Histograms of $(A)$ main phase magnitude (MP) in negative units of Dst, $(B)$ sudden commencement (SC) magnitude in positive units of Dst, $(C)$ the relative difference between the sudden commencement and the main phase, and $(D)$ the time between the sudden commencement and main phase.

3. Cumulative distribution histograms of $(A)$ main phase (MP) magnitude in negative units of Dst, $(B)$ sudden commencement $(\mathrm{SC})$ magnitude in positive units of Dst, $(C)$ the relative difference between the SC and MP, and $(D)$ the time between the SC and MP.

4. Plot showing correlation between main phase magnitude and sudden commencement magnitude................... 6

5. Graph showing overlay of two superposed epoch analyses centered on the storm main phase and sudden commencement (SC) magnitudes.

6. Graph showing a combined average storm profile from the superposed epoch analyses centered on the storm main phase and sudden commencement magnitudes.

7. Scaled storm profiles for small, medium, and large, with each half of the superposed epoch analysis scaled independently.

8. The top panel shows the magnitude of the time rate of change of the U.S. Geological Survey 1-minute Dst index that is shown in the bottom panel.

9. Graph showing the normalized cumulative distribution function for $\mathrm{d}(\mathrm{Dst}) / \mathrm{dt}$ recorded by the U.S. Geological Survey 1-minute Dst during the years 1985-2007. Note the logarithmic scale on both axes........................... 11

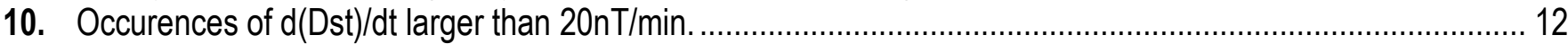

11. Graph showing an overlay of the large storm profile with an actual storm of about the same magnitude......... 13 


\title{
Superposed Epoch Analysis and Storm Statistics from 25 Years of Dst
}

\author{
By J.L. Gannon
}

\begin{abstract}
Statistics on geomagnetic storms with minima below -50 nanoTesla (nT) are compiled using a 25-year span of the 1-minute resolution disturbance index, U.S. Geological Survey Dst. A sudden commencement, main phase minimum, and time between the two has a magnitude of $35 \mathrm{nT},-100 \mathrm{nT}$, and 12 hours, respectively, at the 50th percentile level. The cumulative distribution functions for each of these features are presented. Correlation between sudden commencement magnitude and main phase magnitude is shown to be low. Small, medium, and large storm templates at the 33rd, 50th, and 90th percentile are presented and compared to real examples. In addition, the relative occurrence of rates of change in Dst are presented.
\end{abstract}

\section{Introduction}

There is no such thing as a "typical" geomagnetic storm. Individual geomagnetic storms are as unique as the solar wind enhancements that drive them. High speed streams, corotating interaction regions, and coronal mass ejections all have unique Dst signatures (Richardson, 2006; Denton and others, 2006). Nowhere is this more evident than in the numerous and complicated prediction models that exist (Temerin and Li, 2002; Weimer and others, 2010), which try to quantify how variations in the solar wind produce the variations in the magnetic field measurements on the Earth's surface. Just as numerous are the space weather models that use the Dst index as a driver or proxy for geomagnetic activity (for example, Jacchia-Bowman 2008 ionospheric drag model [Bowman and others, 2008]; Tsyganenko magnetic field models [Tsyganenko and others 1996, 2002a, 2002b, 2005]; Olson-Pfitzer dynamic model [Pfitzer and others, 1988]). In order to use these models for predictive purposes, a prediction of Dst is necessary as an input. Statistical or climatological averages can be used as one form of predictive Dst input.

\section{Data}

In this analysis, storms are selected from the USGS 1-minute Dst index (Gannon and Love, 2010; Gannon and others, 2011). There are other indices that could be used (Sugiura, 1964; Karinen and Mursula, 2006; Love and Gannon, 2009), however, the 1-minute USGS Dst is used in this analysis because the time resolution is high enough to sufficiently resolve a minutes-long storm sudden commencement, while still using low latitude observatories to minimize the effect of auroral electrojet influences on the time series. In addition, the USGS Dst algorithm produces an index with a distribution peak near $0 \mathrm{nT}$ (contrary to the Kyoto method, which is slightly negative). This means that USGS Dst, quiet times on average are near zero, which facilitates the selection criteria. 
The entire data set available at the present time, 1985-2008, is used to select all storms that fit the following criteria:

Dst mininum must be less than $-50 \mathrm{nT}$ at some point during the storm period. This threshold was selected to include as many storms as possible (including small storms).

The storm must have a "classical" shape, with an identifiable main phase. Solitary sudden impulses or broad increases in activity, as might be seen with high speed streams, were not included, even if they dip below the $-50 \mathrm{nT}$ threshold.

The storm interval is selected based on the excursion from, and return to, the background level. On average, this should be a 0nT crossing, but it will vary for individual storms.

The main phase is defined as the point in time when Dst reaches its minimum during the storm interval.

The sudden commencement is defined as the largest Dst maximum preceding the main phase.

Multiphase storms whose individual phase minima reach -50 nT are considered individually.

Figure 1 shows an example of one of the 300 storms selected under these criteria. The main phase (MP), sudden commencement (SC), and storm interval are labeled. All storms are hand selected for this study, but automatic selection rules could also be applied. The storm interval is the most difficult property to define, making it nearly impossible to identify consistently, even by hand. For this reason, only statistics pertaining to the easily identifiable SCs and MPs are analyzed. This study also makes no distinction between storms in different parts of the solar cycle. Different types of storm activity may be more common during different phases of the solar cycle. The possible differences in Dst response because of different types of solar wind enhancement are well beyond the scope of this report. However, data are used from approximately two complete solar cycles, so that any bias because of a dominant activity occurring in a particular solar cycle phase is minimized. $(D)$ 


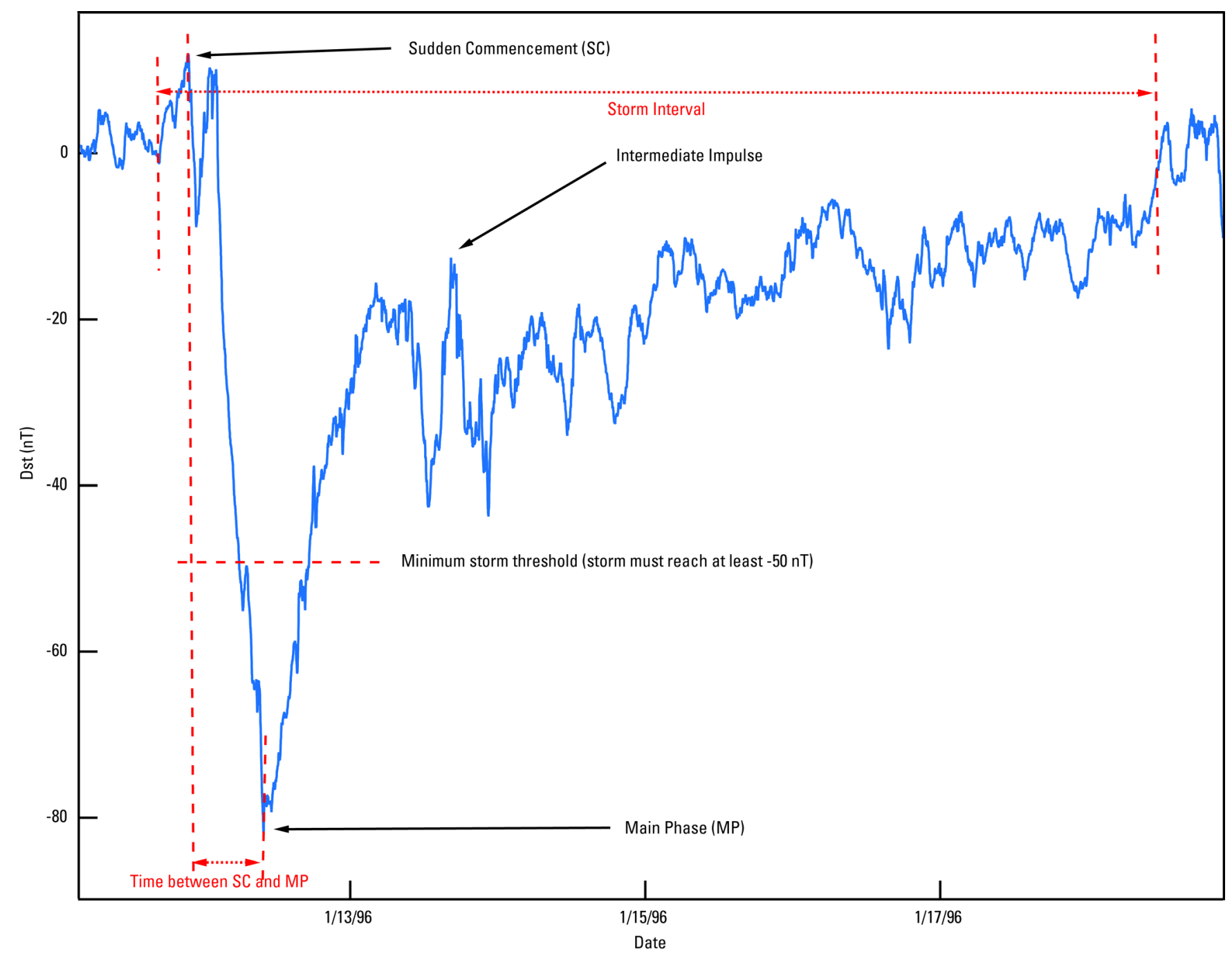

Figure 1. Graph showing an example of a storm period selected under the criteria of this study.

\section{Statistics}

In this study, every storm has a MP, simply defined as the minimum point in Dst. Looking at the histogram of the MP magnitudes for all of the storms in the Dst time series (fig. $2 A$ ), it is evident that smaller storms occur more commonly than larger storms. This is not surprising, and matches well with the observational understanding of geomagnetic activity. The most common storm is in the range of -80 to $-100 \mathrm{nT}$. The cutoff threshold is clearly marked in the histogram as an artificial dropoff at $-50 \mathrm{nT}$.

Not all storms in this study have SCs, which are defined as the maximum Dst value (usually positive, but not always) preceding the previously identified MP. Figure $2 B$ shows the histogram of SC magnitudes for all of the storms in the Dst time series. SC magnitudes or, at least those preceding classical storms reaching at least $-50 \mathrm{nT}$, are more evenly distributed. The most common magnitude in this sample is around $30 \mathrm{nT}$.

There are some caveats to this type of histogram analysis. The "typical" storm should begin at precisely $0 \mathrm{nT}$ (quiet time average), increasing to a maximum value at $\mathrm{SC}$, and then dropping to a 
minimum value during the MP. However, real life is more complicated. The quiet time Dst has a distribution of its own, meaning that some storms will begin at a slightly positive or negative initial value. Because of this, some spread in both SC and MP histograms around the mean is expected. A different way of looking at these data is to consider the difference between the SC and MP magnitudes. Figure $2 C$ shows a histogram of these values. This describes the full range of the storm variation, as the expected physical process might produce, clearly peaking at $100 \mathrm{nT}$ total deviation. However, some information is lost as the actual Dst excursions are not represented.
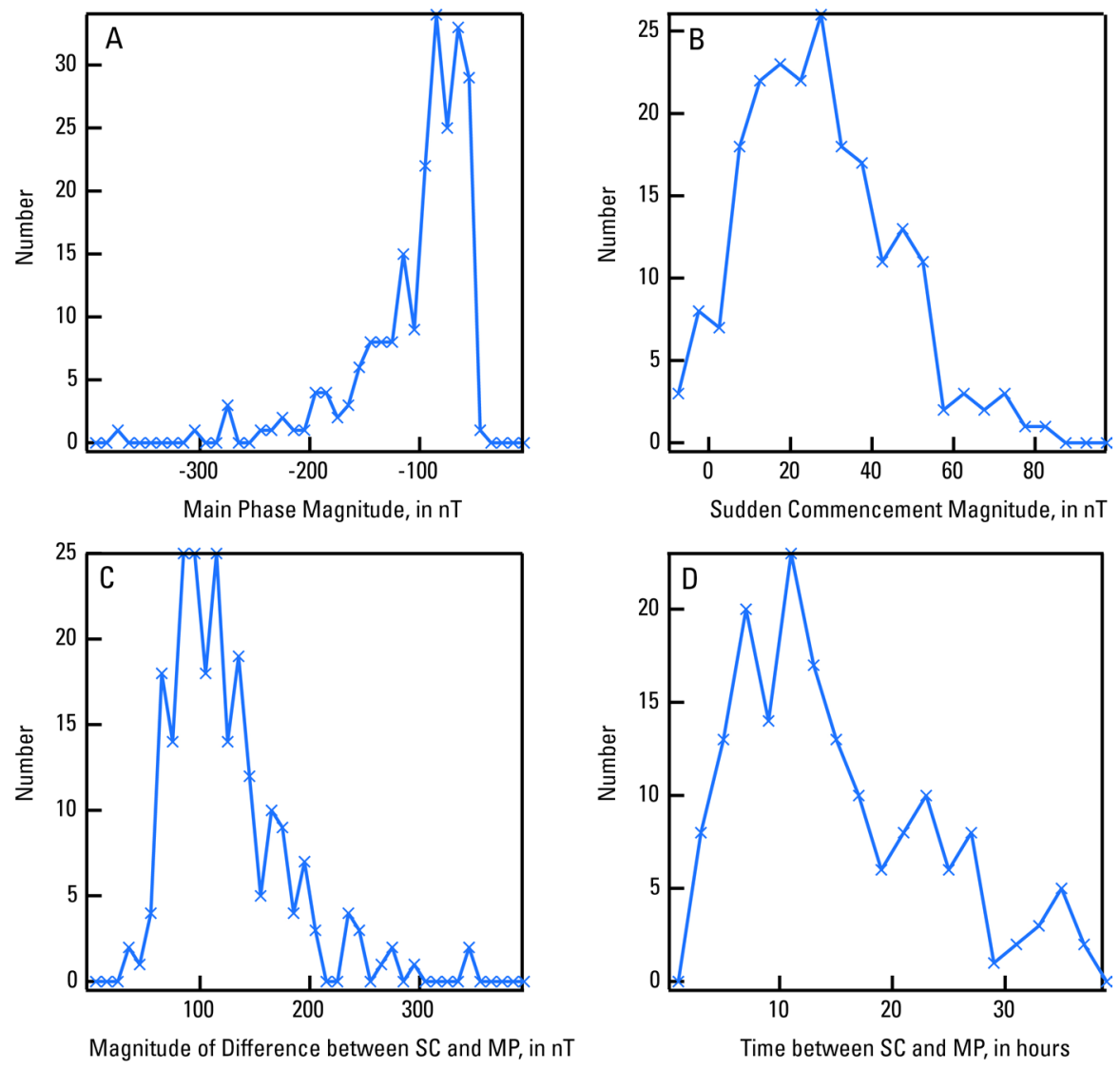

Figure 2. Histograms of $(A)$ main phase magnitude (MP) in negative units of $D s t,(B)$ sudden commencement (SC) magnitude in positive units of Dst, $(C)$ the relative difference between the sudden commencement and the main phase, and $(D)$ the time between the sudden commencement and main phase.

An additional variable needed to describe a storm is the storm duration. Although the storm intervals are somewhat arbitrary and may be identified differently depending on the criteria or algorithm used, the SC and MP are simply and clearly defined features. As a proxy for storm duration, the time it takes to reach minimum Dst during the main phase beginning from the $\mathrm{SC}$ is considered. This minimum reflects a turning point in the physical process, where the magnetic field begins to recover. Figure $2 D$ shows a histogram of these durations, ranging from a few hours to several days. The most common length of time is approximately 12 hours.

To look at the same data in a different way, figure $3 A-D$ shows the same statistics displayed in cumulative $(\mathrm{CDF})$ form. This format allows the relative occurence level of each of these features to be 
assessed. For example, a MP magnitude at the 50th percentile level is approximately -100nT. A SC at the same percentile is about $35 \mathrm{nT}$. Because of the symmetric distribution, this is also the mean value noted from the histogram.
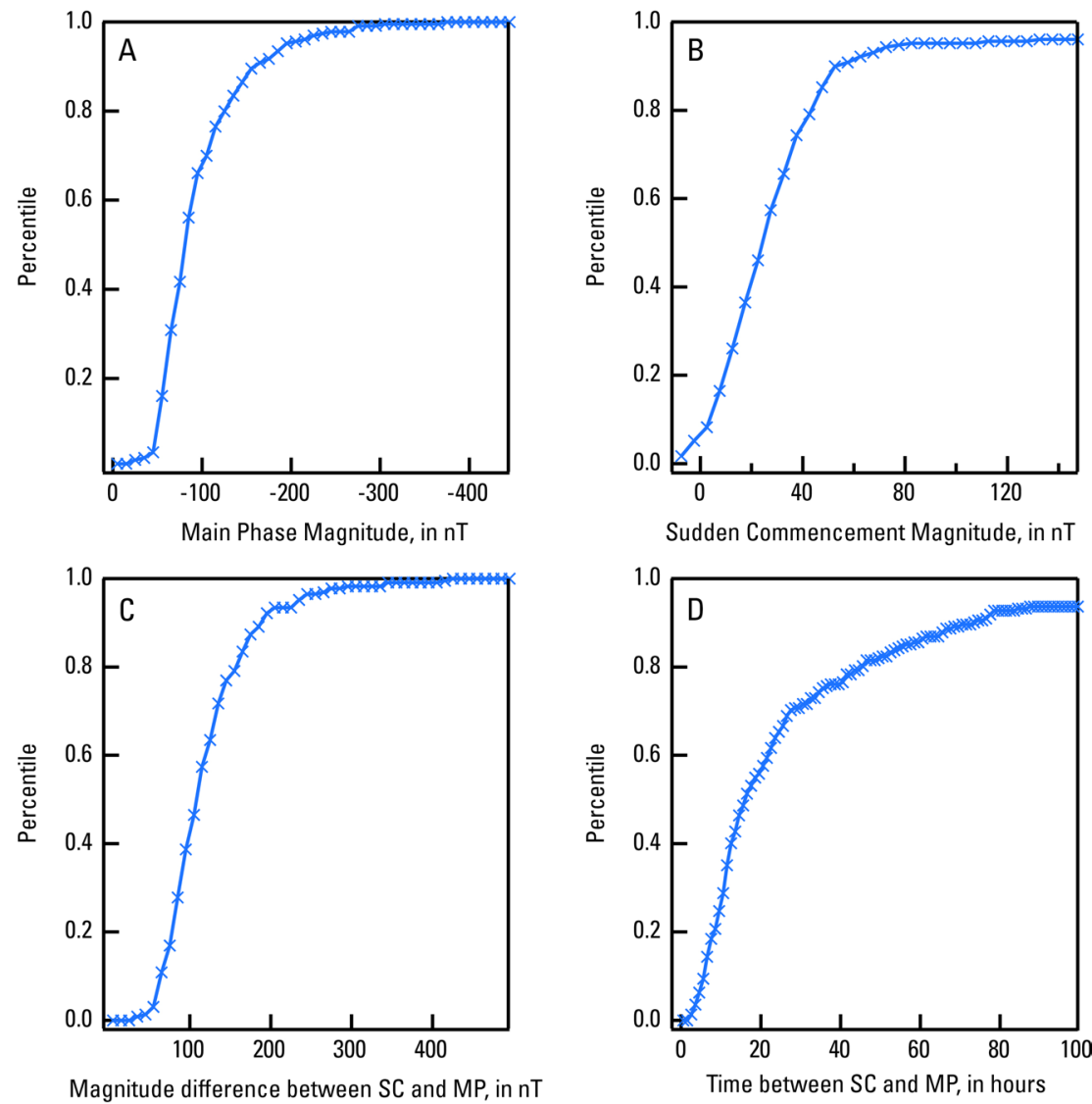

Figure 3. Cumulative distribution histograms of $(A)$ main phase (MP) magnitude in negative units of Dst, $(B)$ sudden commencement $(\mathrm{SC})$ magnitude in positive units of Dst, $(C)$ the relative difference between the $S C$ and MP, and $(D)$ the time between the SC and MP.

\section{"Average" Storm}

From the statistics compiled in the previous section, an idea was formed of what an average storm may look like. A storm in the 50th percentile will have a SC of $35 \mathrm{nT}$, followed by a MP that reaches $-100 \mathrm{nT}$. But how likely is an individual storm with that SC magnitude to have that MP minimum? If the SC and MP magnitudes are highly correlated, this should happen quite often. However, figure 4 shows that the correlation between these values in the dataset is quite low. This is somewhat counterintuitive as the low correlation between SC and MP suggests a high level of independence between two features that are usually considered to be part of the same phenomenon. This makes prediction difficult and complicated. In this analysis, such complications are avoided by describing the statistics of SC and MP magnitude separately. 


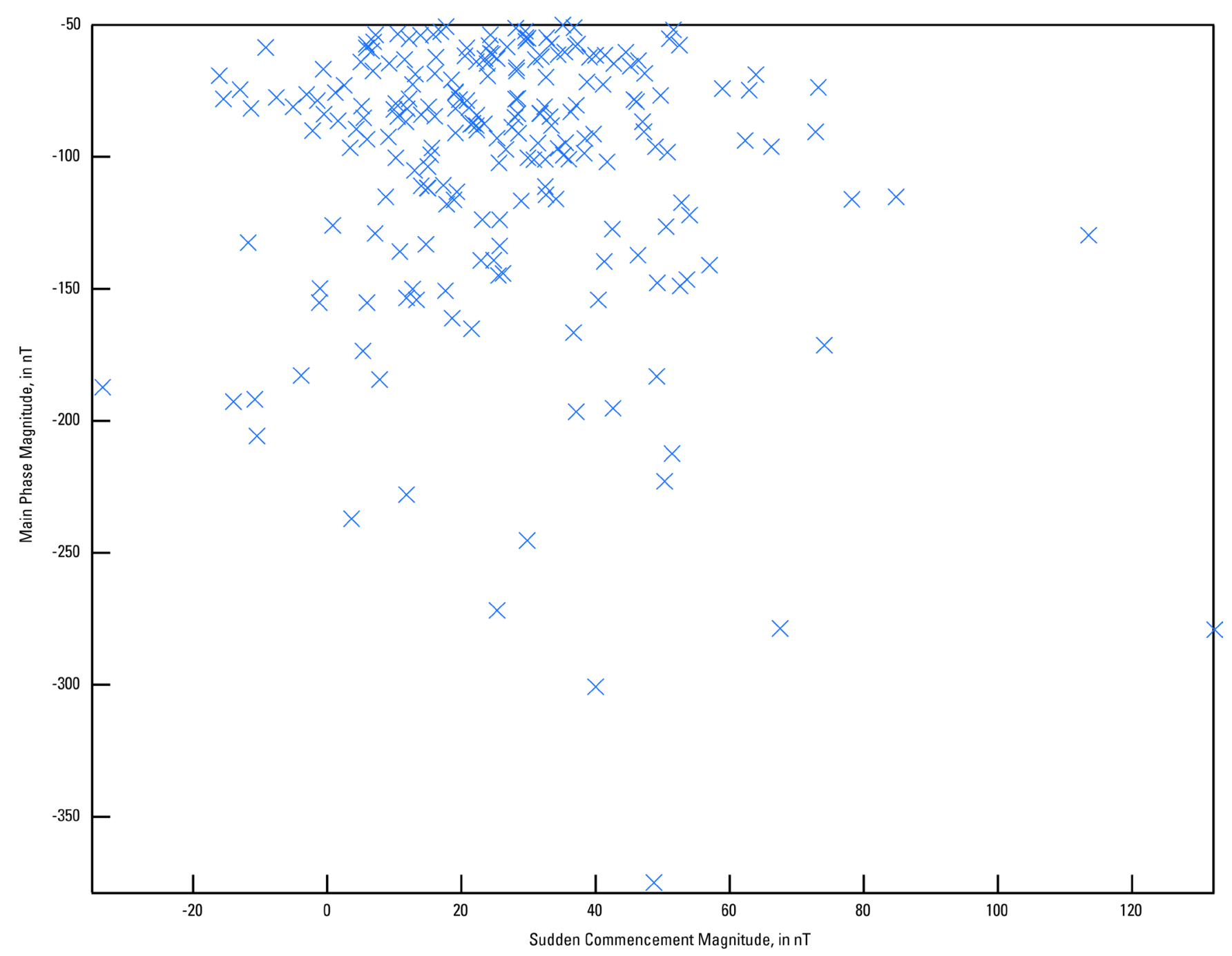

Figure 4. Plot showing correlation between main phase magnitude and sudden commencement magnitude.

To construct an average event, superposed epoch analysis is used, centered on the feature to be explored (called the zero epoch). This is a method of averaging time series data used commonly in the space sciences (Taylor and others, 1994; Hutchinson and others, 2011). In this case, the analysis is centered on the SC and MP separately, averaging all of the storms at each point in time before and after the selected zero epoch. The two resulting average time series are shown overlaid in figure 5 . The blurring of one feature when the average is centered on the other emphasizes that there is a distribution in time between the two features. By centering the average on one, that feature is emphasized and the other is smoothed out. In the overlay, the zero epoch between the two is offset by the average time difference between a SC and MP (12 hours), discussed in the previous section. This gives an average storm shape based on all of the available data (fig. 6). To produce an example of what a storm might look like, the SC and MP can be scaled separately to a specified percentile. Even though the SC and MP are not well-correlated, the 33rd, 50th, and 90th percentiles are chosen for both features to produce small, medium, and large storm templates. The choices of percentile magnitude are arbitrary, as the two phases may be scaled to any level, but are intended to be descriptive of profiles representing low, moderate, and high levels of activity. Figure 7 shows the small, medium, and large templates scaled in this way. 


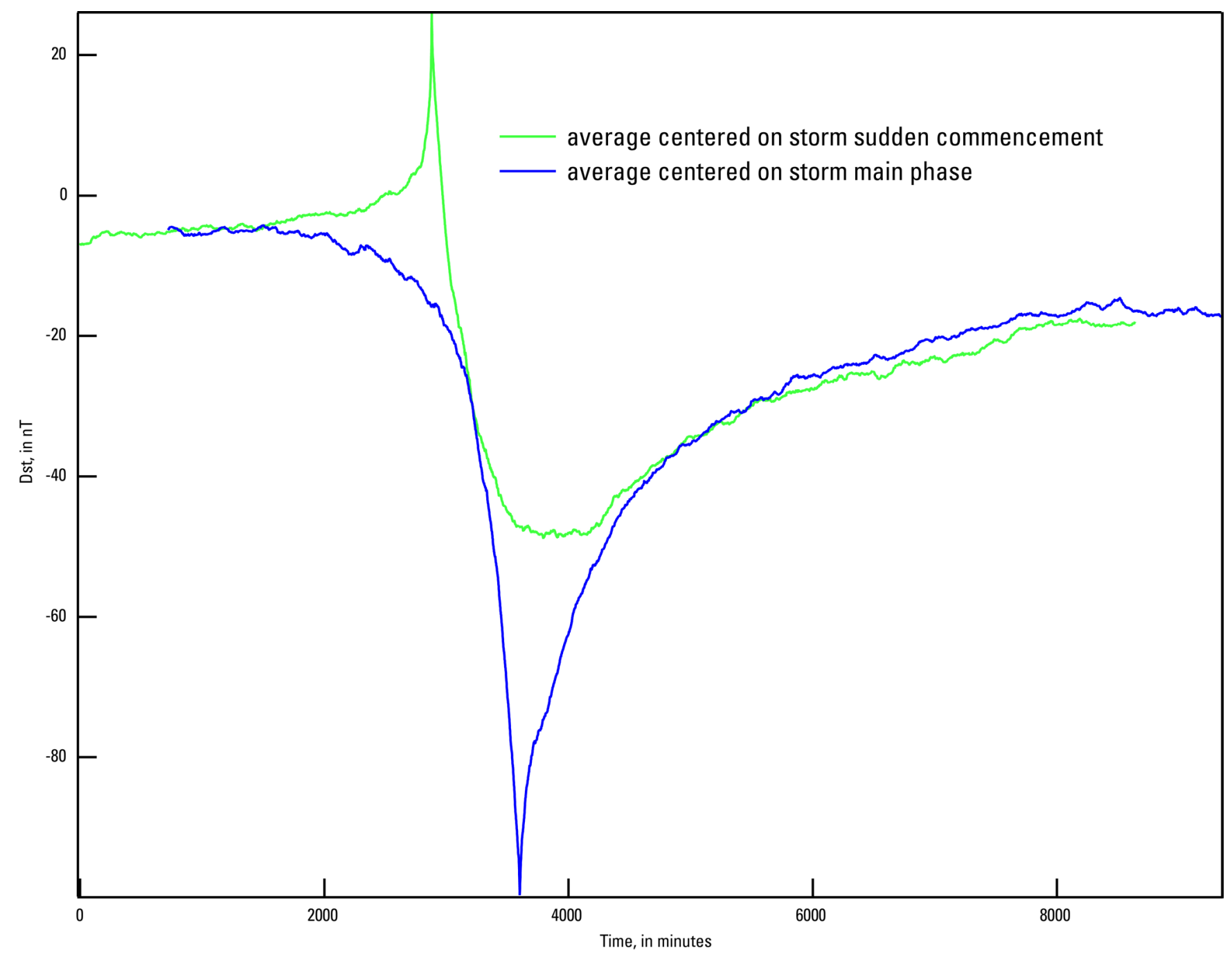

Figure 5. Graph showing overlay of two superposed epoch analyses centered on the storm main phase and sudden commencement (SC) magnitudes. 


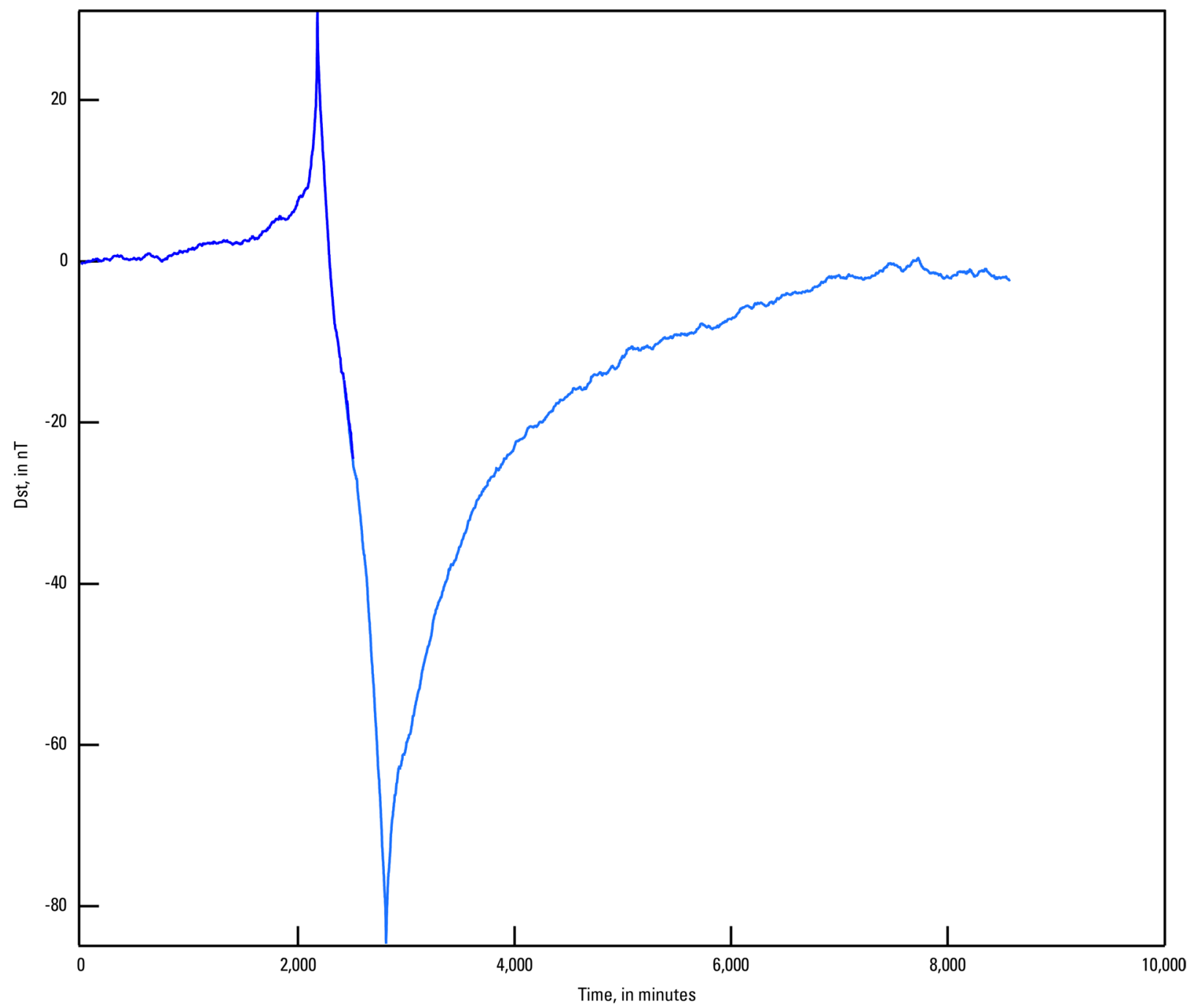

Figure 6. Graph showing a combined average storm profile from the superposed epoch analyses centered on the storm main phase and sudden commencement magnitudes. 


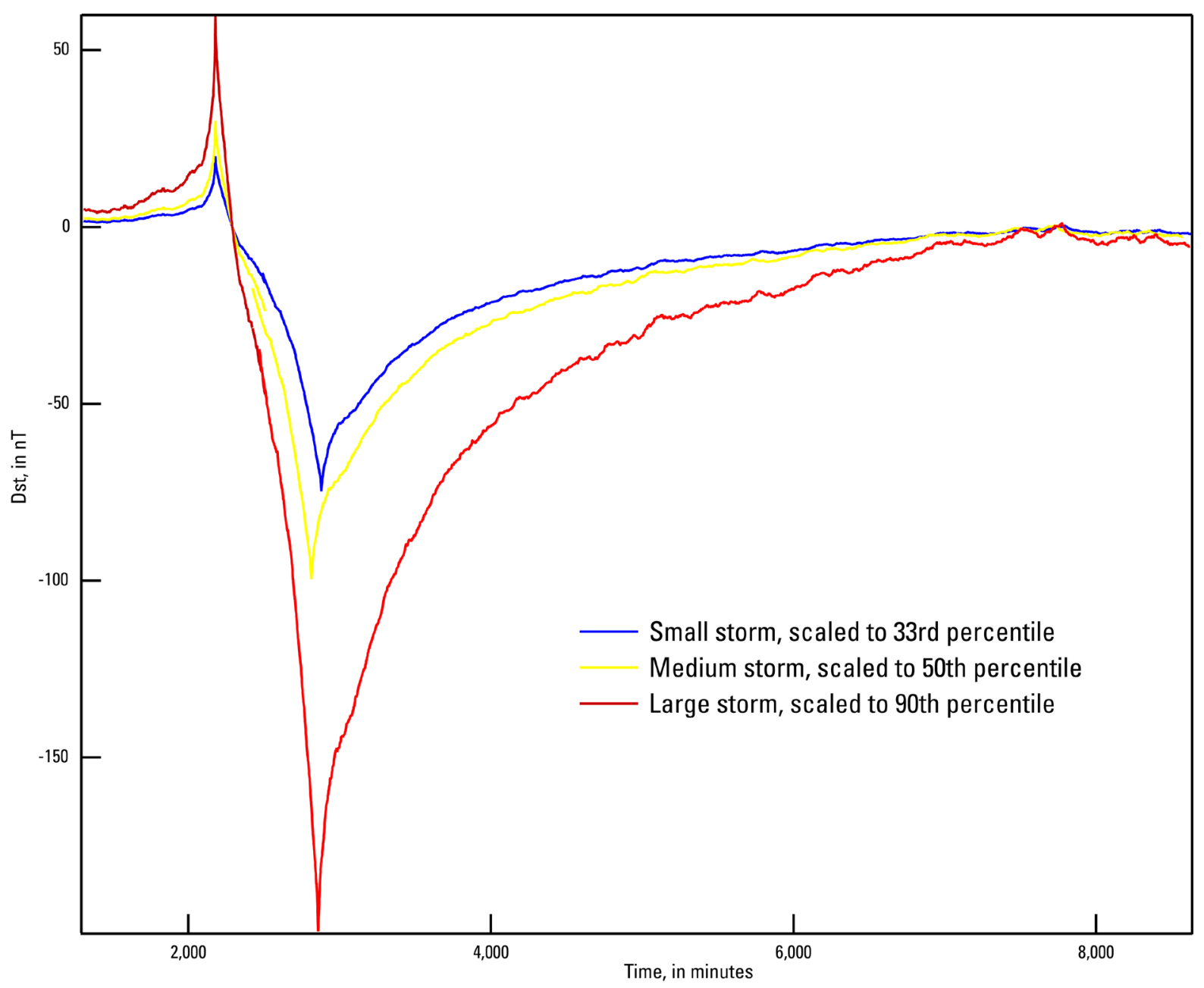

Figure 7. Scaled storm profiles for small, medium, and large, with each half of the superposed epoch analysis scaled independently.

\section{Time Rate of Change of Dst}

The rate of change of the magnetic field with time at a particular location is an important parameter for understanding possible effects of space weather on the ground. Although Dst is a global average and, therefore, does not describe any one specific location, the rate of change of Dst with time can be an indicator for possible local variations. Because this index has a 1-minute time resolution, the time rate of change for rapid effects (for example, sudden impulses and storm SC) can be more precisely quantified than in a traditional 1-hour time resolution storm index. Figure 8 shows Dst and the magnitude of d(Dst)/dt for the USGS 1-minute Dst from the years 1985-2007. The largest spikes are correlated with storm periods; however, the quickest changes do not necessarily correlate with the largest storms. For each of the storm periods analyzed in the previous sections, the maximum $\mathrm{d}(\mathrm{Dst}) / \mathrm{dt}$ is recorded. The normalized CDF in figure 9 shows the relative occurrence of these maxima. From this $\mathrm{CDF}$, it is clear that large $\mathrm{d}(\mathrm{Dst}) / \mathrm{dt}$ are relatively rare. Figure 10 shows the (non-normalized) histogram of those changes larger than $20 \mathrm{nT} / \mathrm{min}$. As changes in local magnetic field get larger with time, induced electric field and geomagnetically-induced currents are enhanced, posing a greater danger to oil pipeline and power grid infrastructure. 


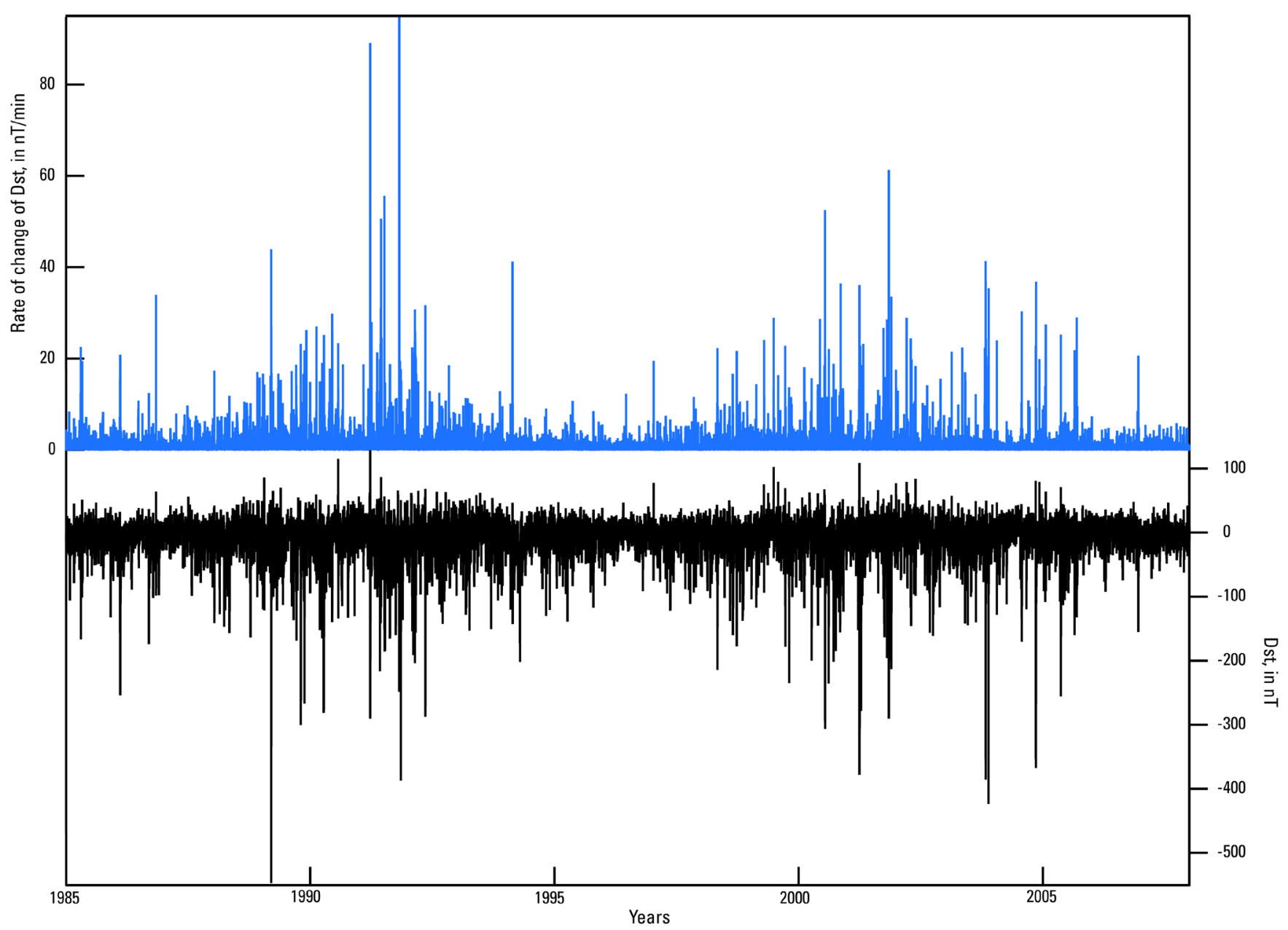

Figure 8. The top panel shows the magnitude of the time rate of change of the U.S. Geological Survey 1-minute Dst index that is shown in the bottom panel.

Figure 11 shows a comparison of an actual storm and the 90th percentile storm profile. There are some timing and magnitude differences at the MP minimum, and the minute to minute variation in the actual storm is much more noisy in comparison to the storm profile, but in general, the basic characteristics match very well. 


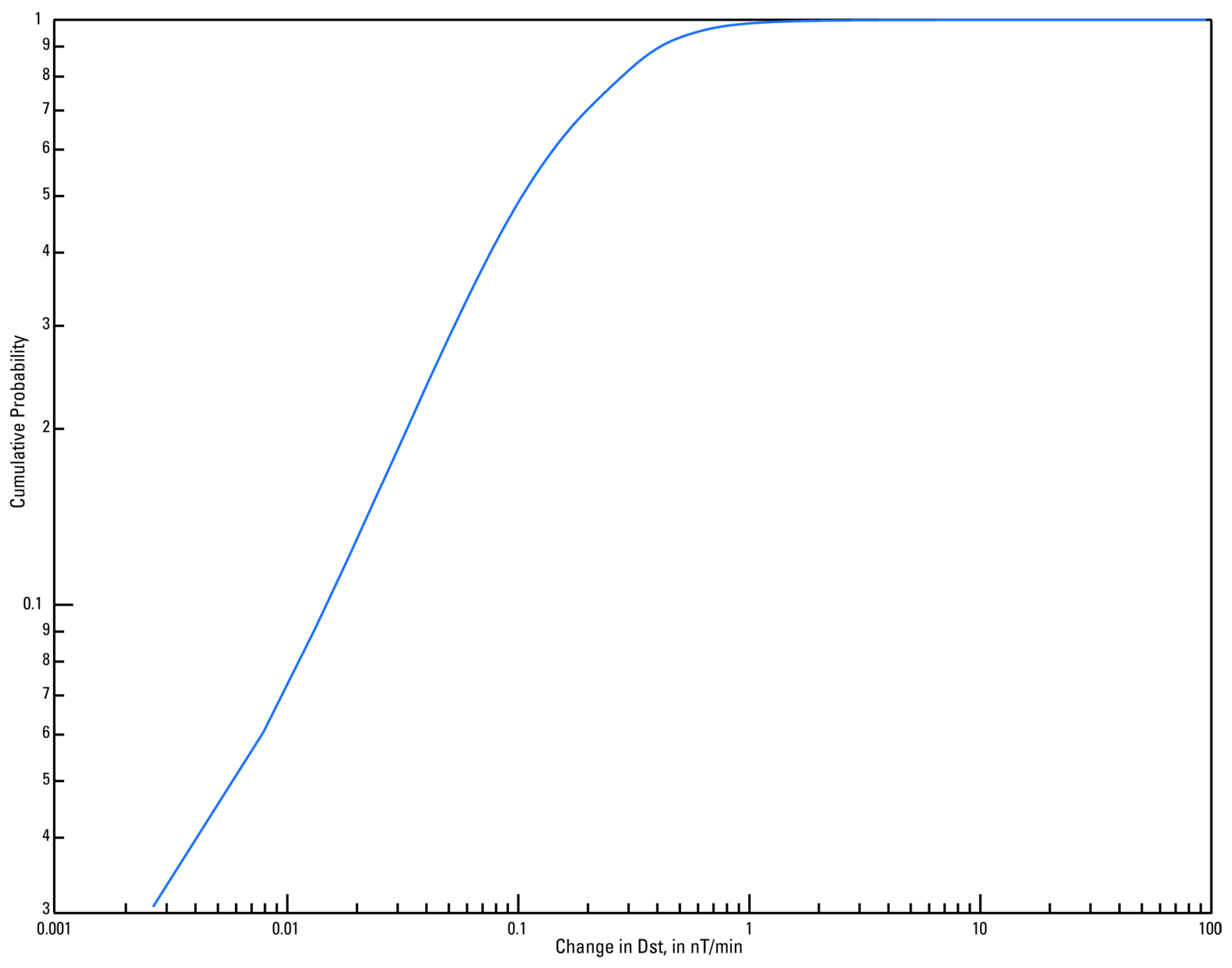

Figure 9. Graph showing the normalized cumulative distribution function for $\mathrm{d}(\mathrm{Dst}) / \mathrm{dt}$ recorded by the U.S. Geological Survey 1-minute Dst during the years 1985-2007. Note the logarithmic scale on both axes 


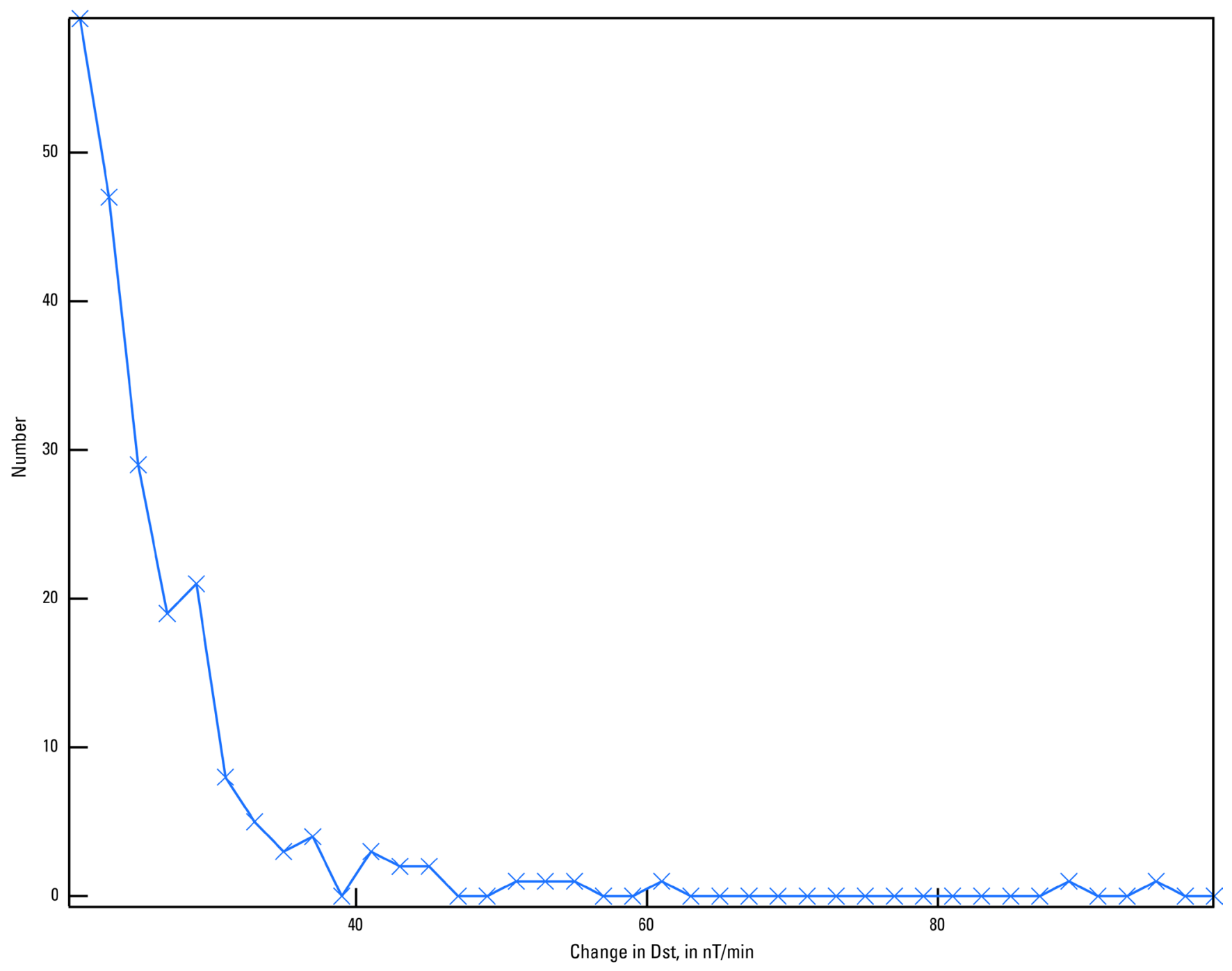

Figure 10. Occurences of $d(D s t) / d t$ larger than $20 \mathrm{nT} / \mathrm{min}$. 


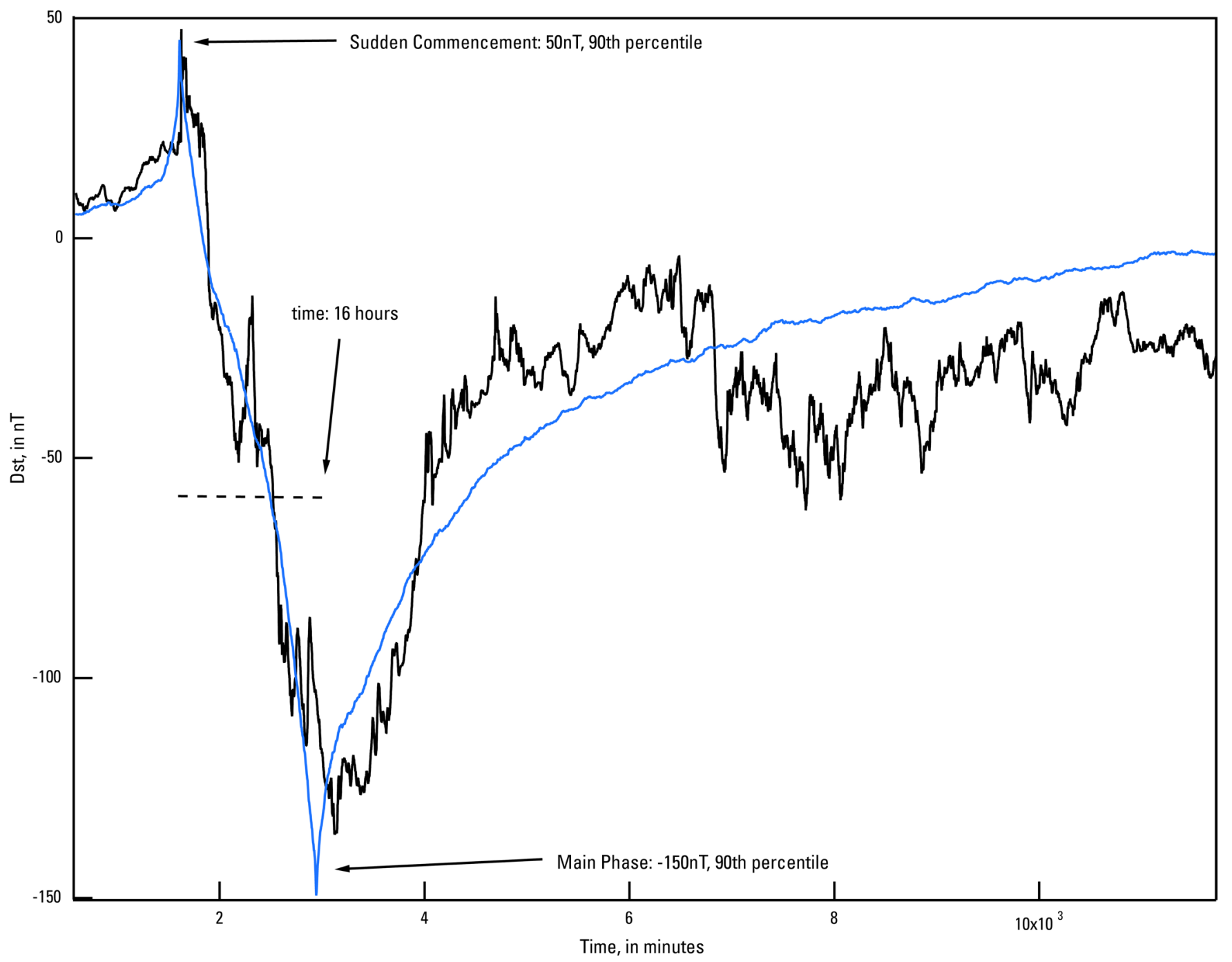

Figure 11. Graph showing an overlay of the large storm profile with an actual storm of about the same magnitude.

\section{Summary}

Three storm templates are developed based on main phase and sudden commencement magnitude statistics. The small, medium, and large templates are specified at the 33rd, 50th, and 90th percentile in magnitude. The statistics of main phase and sudden commencement magnitude are analyzed as normalized histograms and cumulative forms to produce the percentile rating. These are done individually, as the main phase and sudden commencement magnitudes are not highly correlated. In addition, basic statistics of the time rate of change of the U.S. Geological Survey Dst index are analyzed. Even though this is a global index and not necessarily reflective of specific local change, the change in Dst represents the average magnitude of change and can be used as an indicator for possible local variation. These statistics can be used to understand the relative likelihood of storm magnitude and the relative strength of a specific storm in terms of historical perspective. 


\section{Acknowledgments}

The author wishes to thank: H.A. Simpson for his assistance in this project; C.A. Finn, E.J. Rigler, J.J.Love, J. McCollough, E. So and Gavin Hayes for reviewing a draft manuscript.

\section{References}

Bowman, B.R., Tobiska, W.K., Marcos, F.A., Huang, C.Y., Lin, C.S., and Burke, W.J., 2008, A new empirical thermospheric density model JB2008 using new solar and geomagnetic indices in American Institute of Aeronautics and Astronautics/American Astronautical Society Astrodynamics Specialist Conference proceedings: AIAA 2008-6438.

Denton, M.H., Borovsky, J.E., Skoug, R.M., Thomsen, M.F., Lavraud, B., Henderson, M.G., McPherron, R.L., Zhang, J.C., and Liemohn, M.W., 2006, Geomagnetic storms driven by ICME- and CIR-dominated solar wind: Journal of Geophysical Research, v. 111, no. A07S07, doi:10.1029/2005JA011436.

Gannon, J.L., Love, J.J., Friberg, P.A., Stewart, D.C. and Lisowski, S.W., 2011, U.S. Geological Survey near real-time Dst index: U.S. Geological Survey Open-File Report, 2011-1030, 10 p.

Gannon, J.L., and Love, J.J., 2010, USGS 1-min Dst index: Journal of Atmospheric and SolarTerrestrial Physics, v. 73, p. 323-334.

Hutchinson, J.A., Wright, D.M., and Milan, S.E., 2011, Geomagnetic storms over the last solar cycle: A superposed epoch analysis: Journal of Geophysical Research, v. 116, A09211, doi:10.1029/2011JA016463.

Karinen, A., Mursula, K., 2006, A new reconstruction of the Dst index for 1932-2002: Journal of Geophysical Research, v. 11, no. A08207, doi:10.1029/2005JA011299.

Love, J.J., and Gannon, J.L., 2009, Revised Dst and the epicycles of magnetic disturbance: 1958-2007: Annales Geophysicae, v. 27, p. 3101-3131.

Pfitzer, K.A., Olson, W.P., and Mogstad, T., 1988, A time dependent source driven magnetospheric magnetic field model: EOS, v. 69, p. 426

Richardson, I.G., 2006, Major geomagnetic storms (Dst< $-100 \mathrm{nT}$ ) generated by corotating interaction regions: Journal of Geophysical Research, v. 111, A07S09, doi:10.1029/2005JA011476.

Sugiura, M., 1964, Hourly values of equatorial Dst for the IGY: Annals of International Geophysics, Edition 35, p.9-45.

Taylor, J.R., Lester, M., and Yeoman, T.K., 1994, A superposed epoch analysis of geomagnetic storms: Annales Geophysicae, v. 12, p. 612-624, doi:10.1007/s00585-994-0612-4.

Temerin, M., and Li, X., 2002, A new model for the prediction of Dst on the basis of the solar wind: Journal of Geophysical Research, v. 107, no. A12, p. 1,472.

Tsyganenko, N.A., 1996, Effects of the solar wind conditions on the global magnetospheric configuration as deduced from data-based field models, in Proceedings of the 3rd International Conference on Substorms (ICS-3): Versailles, France, European Space Agency, p. 181-185.

Tsyganenko, N.A., 2002a, A model of the near magnetosphere with a dawn-dusk asymmetry: 1. Mathematical structure: Journal of Geophysical Research, v. 107, no. A8, p. 1,179, doi:10.1029/2001JA000219.

Tsyganenko, N.A., 2002b, A model of the near magnetosphere with a dawn-dusk asymmetry: 2. Parameterization and fitting to observations: Journal of Geophysical Research, v. 107, no. A8, p. 1176, doi:10.1029/2001JA000220. 
Tsyganenko, N.A., and Sitnov, M.I., 2005, Modeling the dynamics of the inner magnetosphere during strong geomagnetic storms: Journal of Geophysical Research, v. 110, no. A03208, doi:10.1029/2004JA010798.

Weimer, D.R., Clauer, C.R., Engebretson, M.J., Hansen, T.L., Gleisner, H., Mann, I., and Yumoto, K., 2010, Statistical maps of geomagnetic perturbations as a function of the interplanetary magnetic field: Journal of Geophysical Research, v. 115, no. A10320, doi:10.1029/2010JA015540. 